\title{
A dual confocal aperturing microscope for IR microspectrometry
}

\author{
D.W. Schiering, T.J. Tague, Jr., J.A. Reffner ${ }^{1}$ and S.H. Vogel \\ Spectra-Tech, Inc., 2 Research Dr., Shelton, CT 06484 USA \\ ${ }^{1}$ Current address: Trace Consulting, 97 Ocean Dr. East, Stamford, CT 06902
}

The design of a reflecting microscope for FT-IR microspectrometry is described. The microscope employs infinity-corrected optics and a novel dual confocal aperturing configuration. The performance of the microscope is discussed relative to stray light rejection and the use of differential interference contrast with IR microspectroscopy.

\section{Introduction}

Fourier transform infrared (FT-IR) microspectroscopy is now almost universally accomplished with the reflecting microscope. Due to the Fellgett (or multiplex) advantage of FTIR instruments and sensitive, photoconductive detectors which they may employ, the measurement of IR spectra of micrometer sized specimens can be made in very reasonable timeframes. The microscope allows precise positioning and measurement area definition as well as providing a magnified image of specimen. The industrial applications of FTIR microspectrometry are quite diverse and include the identification of contaminants in paper [1], polymer films [2], and on the surface of circuit boards [3]. The technique has also been applied to the analysis of semiconductors [4], single fibers [5-7], trace evidence [8,9], antiquities [10], geological samples [11,12], and biological matrices [13,14].
FT-IR microscopes employ at least one masking aperture in the IR optical system. This aperture can be sized to correspond to the sample size and is used to define the sampled area. The aperture is located at a conjugate image plane relative the specimen and therefore, can also be viewed at the same time as the sample. It is well known that the implementation of dual apertures at confocal image planes provides higher spatial resolution and photometric accuracy than single aperture systems [15-17]. In dual aperturing systems, the diffraction-induced spurious signal is reduced by illuminating the specimen with a field corresponding to its size using the first aperture and further reducing the intensity of the side lobes of the diffracted energy spread function by using a second aperture. The dual remote aperturing (or Redundant ${ }^{\mathrm{TM}}$ aperturing) system formed the basis of a generation of FT-IR microscope products [18].

The capabilities of the FT-IR microscope for the observation of specimens should not be overlooked. The images that the microscope generates are important information concerning the sample. FT-IR microscopes vary in the optical microscope capabilities that are provided. Some systems provide nothing more than simple illumination and viewing in addition to the all reflecting microscope optics essential to the IR measurement. Other FT-IR microscope systems provide a nosepiece for multiple objective mounting, refractive objectives, Koehler illumination systems, polarized light illumination, etc.

This report concerns the design of a new reflecting microscope for FT-IR microspectrometry. The general design features have been briefly described [19]. The microscope employs an infinity corrected optical system. Infinity 
corrected microscopes are the current state of the art in optical microscopy and offer versatility not afforded in finite conjugate systems. The IR optical configuration of the microscope will be discussed. The microscope employs a single aperture in a dual pass configuration. This novel approach to dual confocal aperturing provides ease-of-use while maintaining the microspectrometry performance advantages. The performance of this optical system will be demonstrated relative to stray light reduction. This FT-IR microscope design also allows the use of optical contrasting techniques. A description and example use of differential interference contrast (DIC) will be discussed.

\section{Experimental}

The microscope optics used in this study form the basis of the Continuum FT-IR microscope products offered by Nicolet Instruments Corporation (Madison, WI). The IR optical design of the microscope is described in detail below and is the subject of a US Patent [20]. All IR data were measured with a Nicolet Magna 560 FT-IR spectrometer. A ceramic source, $\mathrm{KBr}$ substrate beamsplitter, and a microscope-mounted mercury-cadmium-telluride (MCT) detector were used. The detector had an active area of $250 \times 250 \mu \mathrm{m}$ and an operating temperature of $77 \mathrm{~K}$. An automated aperture placed at a conjugate sample image plane was used to define the measured sample dimension. A Schwarzschild reflecting objective and condenser pair (15X magnification, N.A. $=0.58)$ were used. 50-watt quartz-halogen, Koehler illuminators were used in transmission and reflection modes. Sample positioning was accomplished with a $2 \times 3$ inch travel Olympus ${ }^{\circledR}$ (Lake Success, NY) microscope stage. The microscope and spectrometer were purged with dry air to minimize the infrared absorption of atmospheric water and carbon dioxide.

An IR $\mu s^{T M}$ (Spectra-Tech, Inc., Shelton, CT) microspectrometer was used to record the spectrum of Kevlar ${ }^{\circledR}$ fiber for comparison purposes. A single masking aperture placed after the sample was used in the measurement of the fiber spectrum.

Unless specifically noted otherwise, spectral data were recorded at $4 \mathrm{~cm}^{-1}$ spectral resolution using a triangular apodization function. Double-sided interferograms were recorded at an optical velocity of $1.89 \mathrm{~cm} / \mathrm{sec}$. The number of averaged scans was varied depending upon the experiment. A Mertz phase correction was used in the calculation of the spectra.

$\operatorname{Kevlar}^{\circledR}$ (poly-paraphenylene terephthalamide) fibers (E.I. du Pont de Nemours, Wilmington, DE) were used to evaluate the photometric accuracy of the system. Rat retinas were obtained from the eyes of rats which had been anesthetized and sacrificed. The rat retina cross-section was prepared via frozen sectioning using a cryo-microtome and placed on a $\mathrm{BaF}_{2}$ window for IR analysis. The cross-section was $10 \mu \mathrm{m}$ in thickness.

\section{Results and discussion}

The reflecting microscope described in this work differs from previous designs in three major aspects - the implementation of infinity corrected optics, the use of dichroics to allow the collection of IR data while simultaneously observing the specimen with the optical microscope, and the implementation of dual confocal aperturing using a single mask.

Figure 1 contrasts infinity corrected optics with optics employing finite conjugate image planes, relative to the microscope objective. The finite conjugate optical design is typical in IR microscopes. In this configuration, the objective forms an image of the specimen some distance from the objective pupil. In the infinity-corrected design, the objective collimates the light, i.e. forms an image at infinity, at the back pupil of the objective. A tele-lens in the optical train then forms an image of the specimen for relay to the viewing means. The infinity corrected optical design has become the standard for light microscopes used in research applications. The infinity-corrected design offers several advantages for use in IR microscopes. The design allows the use of standard optical microscope viewers, objectives, and condensers. The implementation of ancillary optical modules is greatly simplified through interface in the parallel light path. These optical modules include image contrasting optics such as polarizers or differential interference contrast prisms, or the dichroic optics described in this report. The infinitycorrected design allows the use of flat mirror relay optics in the IR beam path. The alignment and stability of the system is improved through the use of flat mirror optics.

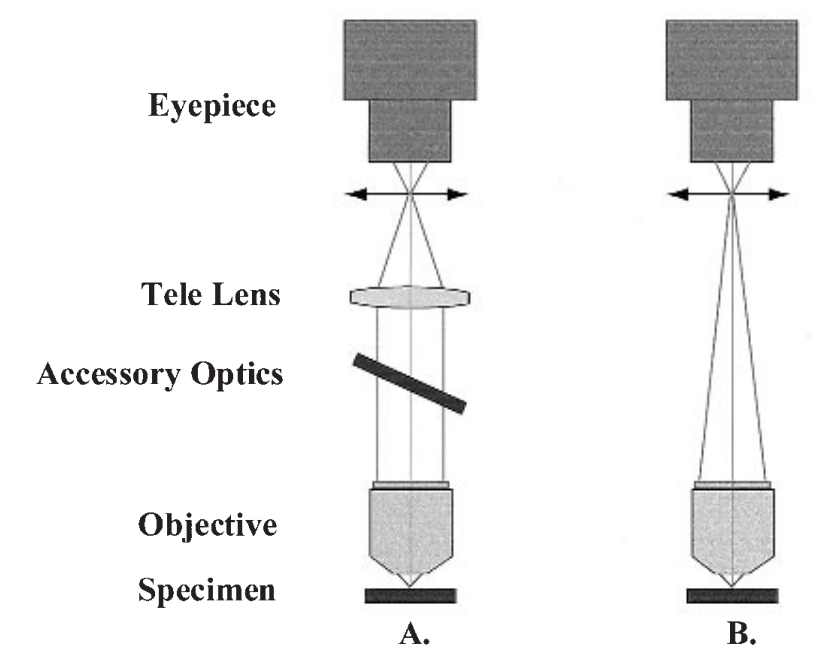

Figure 1. A. Infinity-corrected microscope. B. Finite conjugate microscope. 
Figure 2 shows the IR ray path of the microscope, operated in transmission mode. The collimated output from the FT-IR is intercepted by a flat mirror and directed to a $200 \mathrm{~mm}$ focal length focussing mirror, M1. This optic forms an image of the field stop at the variable masking aperture. The beam is recollimated by a second $200 \mathrm{~mm}$ focal length focussing mirror, M2. A flat mirror, FM1, relays the beam to a dichroic mirror pair, DM1 and DM2. A second image of the field stop is formed at the specimen plane by an infinity corrected, Schwarzschild objective, C1. The transmitted light from the specimen is recollimated by a matched, infinity corrected Schwarzschild condenser, $\mathrm{C} 2$, and relayed by a flat mirror, FM2, and a third dichroic mirror, DM3. A flat relay mirror, FM3, directs the beam to a third $200 \mathrm{~mm}$ focal length focussing mirror, M3. M3 reforms a field stop image at the masking aperture position. After passing through the aperture a second time, the light is recollimated by a fourth $200 \mathrm{~mm}$ focussing optic, M4, and relayed to the detection optics via a flat, dichroic mirror DM4.

The dichroic mirror optics are placed at intersection points of the visible illumination and viewing systems, which are not indicated in figure 2 . The reflection illumination system and viewing system are input to the optical system at dichroic mirror pair DM1 and DM2. The transmission illumination is input at DM3. Masking aperture illumination is input at DM4. These dichroic mirrors are transmissive to visible light (hence their placement at visible light intersects) but highly reflective to IR radiation. Therefore, these optics remain in the IR beam path and the specimen may be viewed at the same time that IR data is collected.

The microscope is operated in a reflection IR mode through insertion of a double-sided splitting mirror between the source input and M1. The mirror is aperture splitting. Half of the optics are used for the incident light and half for the reflected light. The theoretical performance of reflectance IR microspectroscopy is therefore $50 \%$ of transmittance.

The accuracy of the observed transmittances of IR spectra of specimens measured with FT-IR microscopes is known to be affected by stray light [5] induced by diffraction and

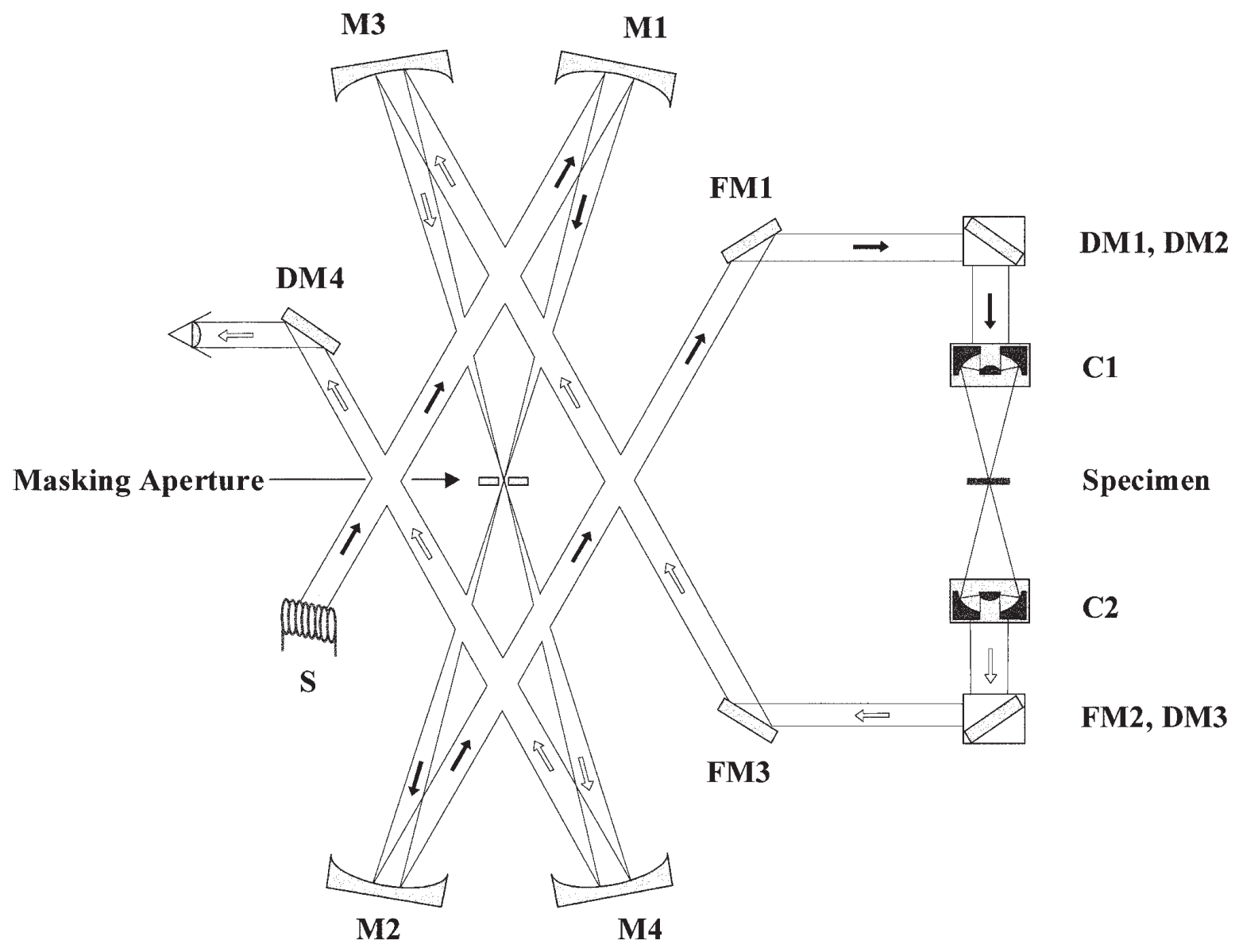

Figure 2. Transmission IR ray diagram of a dual confocal aperturing, reflecting microscope. 
optical aberrations with blur circles larger than diffraction. Due to the fact that the masking aperture is at a magnified, remote conjugate image plane relative to the sample, light in the specimen plane outside the area delineated by the aperture can pass through to the detector. This light does not contain information about the sample. For a "free-standing" sample, e.g. a sample on a transmissive or reflective substrate, this stray light distorts the transmittance of the absorption bands. The photometric accuracy of the data is significantly worsened and the quantitative potential of the technique is lost. Diffraction effects are perhaps even more troublesome in FT-IR microscope measurements when attempting to resolve more than one material. In this case, absorption bands can be observed which are due to a phase outside the masked area. These spurious absorption bands can be easily misinterpreted as sample absorption bands. Several researchers have characterized the general design and performance attributes of FT-IR microscopes relative to stray light resulting from diffraction [15-17]. The magnitude of the distortion that diffraction will cause is dependent upon the wavelength of light and the sample dimension. Messerschmidt demonstrated that stray radiation could be significantly reduced in spectra measured with FT-IR microscopes by employing dual confocal masking apertures, one before and one after the sample $[15,16]$.

Figure 3 illustrates the photometric performance that is possible using the unique type of sample masking system described above and illustrated in figure 2. In this experiment, the spectrum of a monofilament, polymeric sample was measured. The fiber material was $\operatorname{Kevlar}^{\circledR}$ (poly-paraphenylene terephthalamide), selected due to the small filament diameter and the fact that this sample has been previously studied [21]. The diameter of the fiber was $12 \mu \mathrm{m}$, corresponding to $800 \mathrm{~cm}^{-1}$ radiation. The masking aperture (see Fig. 2, above) of the system was sized to match the fiber diameter dimension and corresponded to $100 \mu \mathrm{m}$ along the fiber axis. At $12 \mu \mathrm{m}$ thickness, the $\operatorname{Kevlar}^{\circledR}$ aramide absorption band near $1660 \mathrm{~cm}^{-1}$ would be totally absorbing $(0 \% \mathrm{~T})$, in the absence of diffraction-induced stray light and other optical or electronic imperfections. Using this new dual confocal aperturing configuration, the transmittance of the aramide bands are approximately $4 \% \mathrm{~T}$. This result is consistent with the photometric performance observed on FT-IR microscopes employing separate dual sample masks. Also shown in figure 3 is a spectrum of the same Kevlar ${ }^{\circledR}$ filament measured using a single aperture after the sample. An IR $\mu s^{\mathrm{TM}}$ microspectrometer was used in a single aperture mode for this measurement. The transmittances of the strongest bands are distorted $25-30 \% \mathrm{~T}$.

The infinity corrected optical design also provides for ease in introducing accessory optical modules that are useful for specific applications. Differential interference contrast (DIC) is a technique broadly used in the light microscopy field to enhance contrast of otherwise featureless specimens. The technique is used in the biological, materials science, and semiconductor fields. The microscope described above is the first FT-IR microspectroscopy system to permit the observation of DIC images and the results

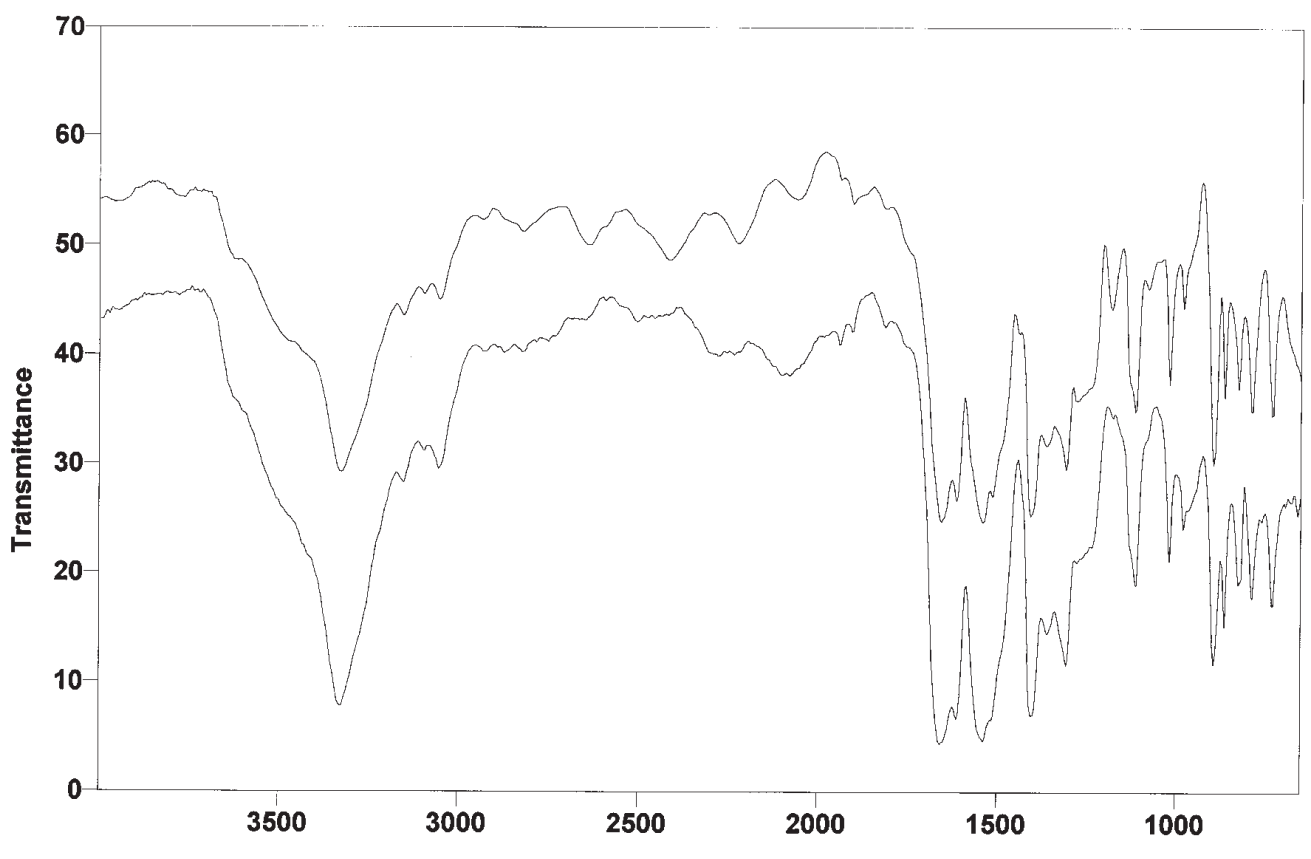

Wavenumber (cm-1)
Figure 3. Kevlar $^{\circledR}$ single fiber spectra. A. Single aperture after sample. B. Single aperture, dual pass. 
shown in this paper are the first reported DIC images using reflecting, Schwarzschild objective optics. Figure 4 illustrates the components required for the generation of DIC images. The light from a visible illuminator is plane polarized. The polarized visible light is introduced to a Wollaston prism. The Wollaston prism generates two orthogonally polarized rays, separated in space. The polarized rays are focussed at the sample by a condenser, in this case a Schwarzschild reflecting optic. The sample then may interact differently with these polarized rays, inducing a phase lag. The phase retarded components interfere and give rise to a three-dimensional shadowing effect, the basis for the contrast. After passing through the sample and the objective, the light is recombined at a second Wollaston prism, and passes through an analyzer. The polarization plane of the analyzer is oriented orthogonally to the first polarizer and ensures that only light that has interacted with the sample will be observed. After the analyzer, the light is passed to the viewing system.

In figure 5 are photomicrographs of a cross section of the retina from a rat. The left image was recorded with conventional, transmitted, white light illumination. The right image was recorded using transmitted light DIC. The retina tissue cross section is oriented diagonally across the center of the field of view in both images. The DIC enhanced image highlights features of the tissue that are not observed in the white light image. In particular, the cellular layered structure of the retina is observed in the DIC image but is not evident in the white light image. The photoreceptor outer segment appears as a yellow layer diagonally across the field of view. In this region of the retina, the chemistry of vision is initiated.

Figure 6 shows two IR spectra recorded from the rat retina tissue section. The top spectrum was recorded from the photoreceptor outer segment. The bottom spectrum was recorded from the outer nuclear layer which is $\sim 60 \mu \mathrm{m}$ along a diagonal from the photoreceptor outer segment towards the center of the DIC photomicrograph in figure 6. The sampled area in both cases was $10 \times 60 \mu \mathrm{m}$ as defined by the masking aperture. The long axis of the aperture was oriented parallel to the cellular layers evident in the DIC image. The difference in the chemical constituency between the photoreceptor outer segment and the outer nuclear layer is evident from comparison of these data. The photoreceptor outer segment has a significant fat component. The increased absorption intensities at $2923 \mathrm{~cm}^{-1}\left(\mathrm{~V}_{\mathrm{as}} \mathrm{CH},-\mathrm{CH}_{2}-\right)$, $1735 \mathrm{~cm}^{-1}(\mathrm{vC}=\mathrm{O})$, and $1456 \mathrm{~cm}^{-1}\left(\delta_{\mathrm{as}} \mathrm{CH},-\mathrm{CH}_{2^{-}}\right)$, are indicative of fatty acid ester moieties. The $3013 \mathrm{~cm}^{-1}(\mathrm{vCH}$, $-\mathrm{C}=\mathrm{CH}-)$ band indicates that the fatty acid ester moieties are at least partially unsaturated. Other differences in composition can also be noted. The strong absorption at $1080 \mathrm{~cm}^{-1}$ $(\mathrm{vC}-\mathrm{O},-\mathrm{COH})$ indicates an increased level of polysaccharides and the absorption at $1250 \mathrm{~cm}^{-1}(\mathrm{vP}=\mathrm{O})$ indicates an increased level of phosphate. The increased absorption intensities of these functional group frequencies are likely due to the higher DNA content of the outer nuclear layer.

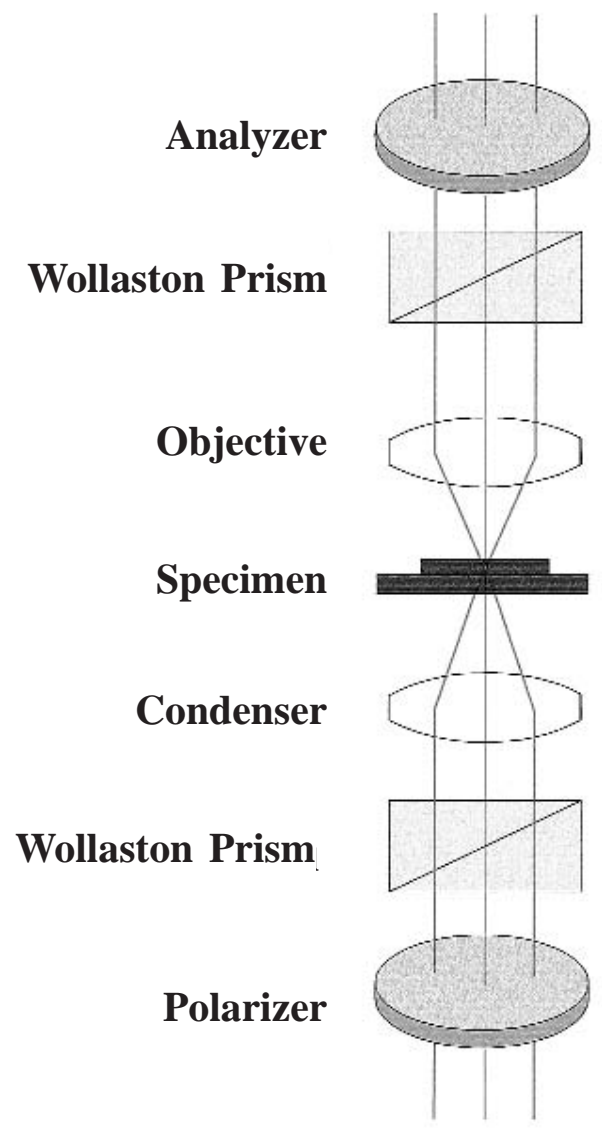

Figure 4. Optics for differential interference contrast.

\section{Conclusion}

A new reflecting microscope design for IR microspectrometry was described. The new design incorporates infinitycorrected optics, a dual-pass masking aperture, and dichroics. The measured stray light at $1660 \mathrm{~cm}^{-1}$ was $\sim 4 \% \mathrm{~T}$ using a $12 \mu \mathrm{m}$ Kevlar $^{\circledR}$ single filament as an evaluation sample. For comparison, the stray light was 25$30 \% \mathrm{~T}$ using a single aperture after the sample. Differential interference contrasting (DIC) was described and demonstrated with a rat retina cross-section. DIC is an optical contrasting technique that will aid in differentiating sample areas for IR measurement in images that are featureless using conventional illumination.

\section{Acknowledgements}

The authors acknowledge Dr. David Wetzel of Kansas State University and Dr. Steven LeVine of the University of Kansas Medical Center for the gift of the rat retina crosssection and helpful discussions. 


\section{Dossier}

A

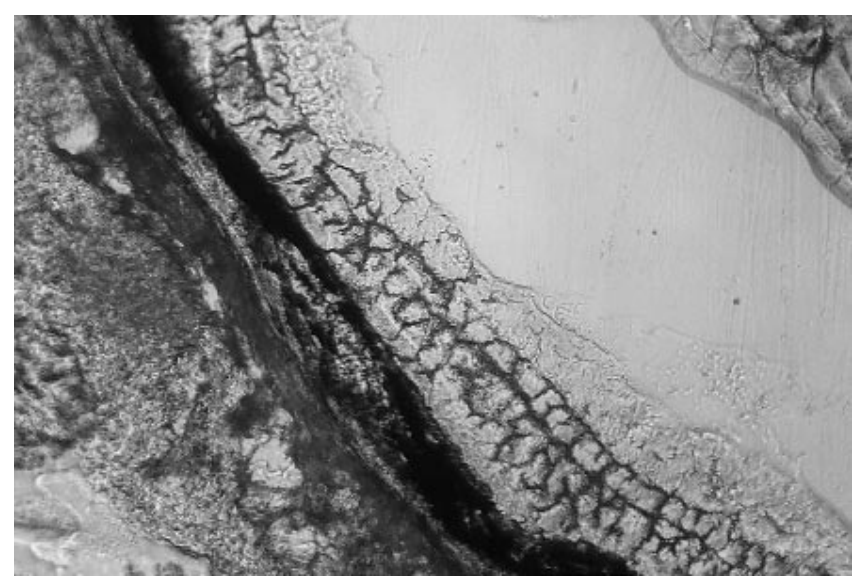

B

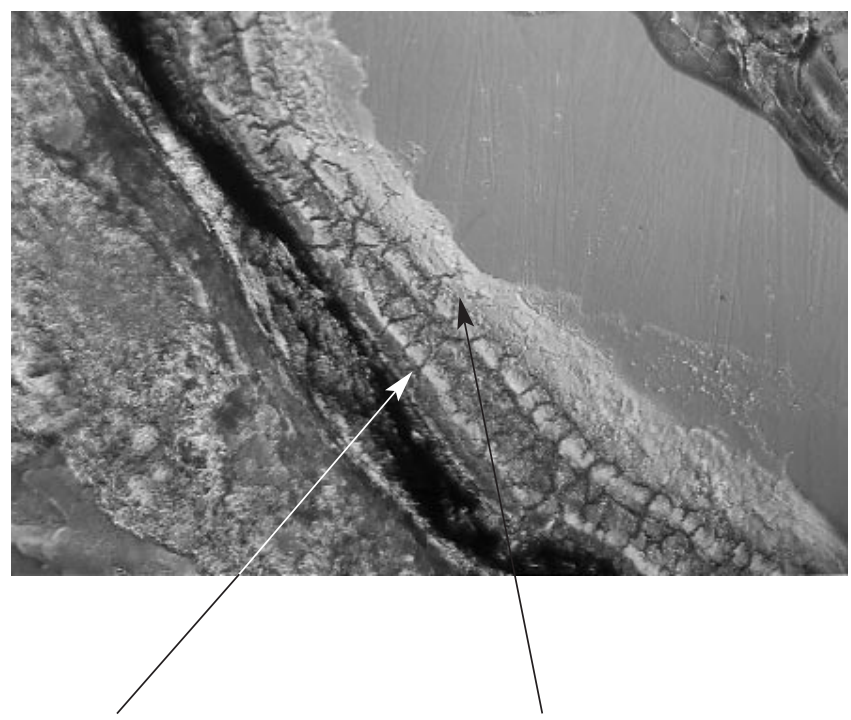

Outer nuclear layer

Figure 5. Rat retina cross-section. A. Transmitted light illumination. B. Transmitted light DIC.

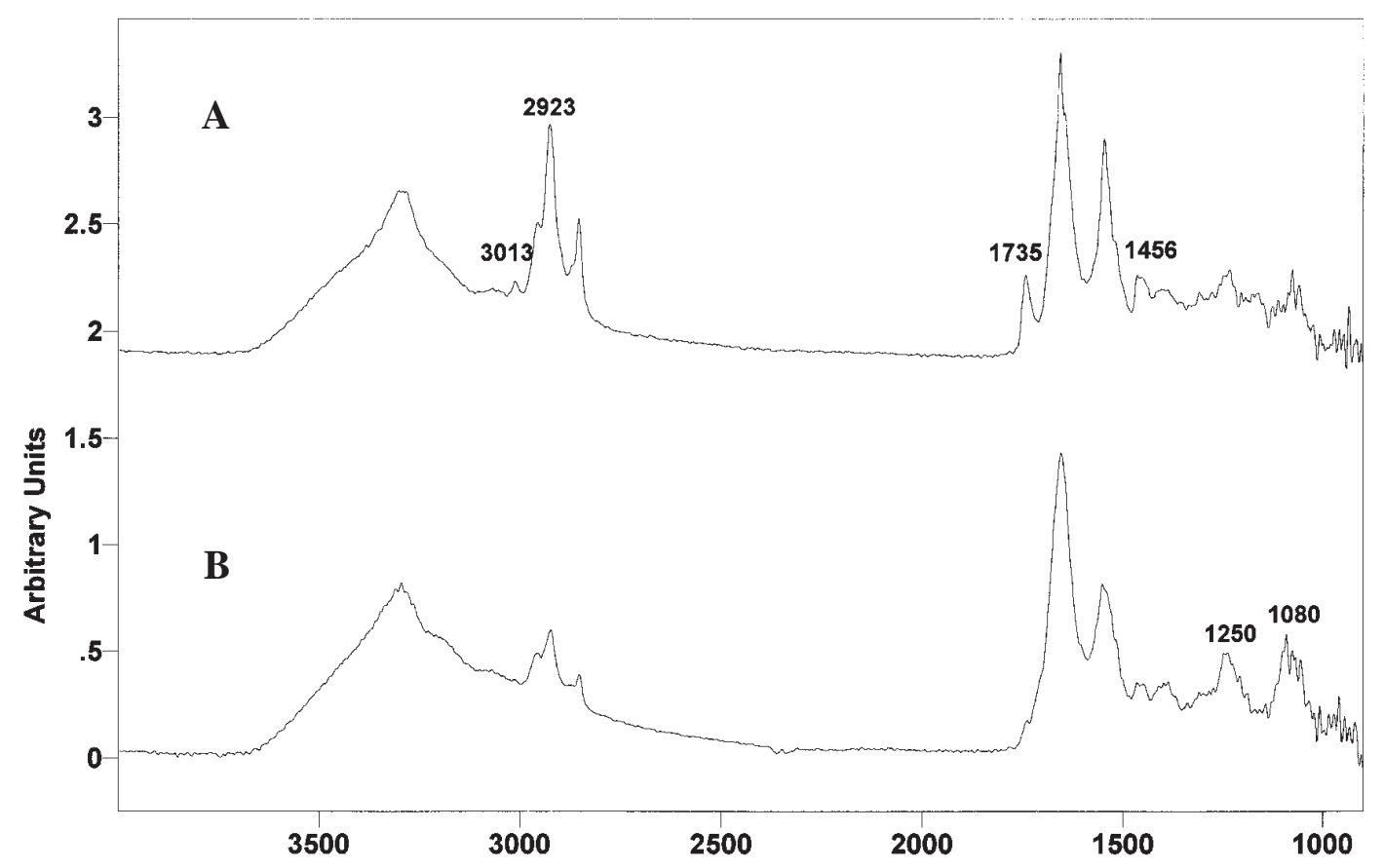

Wavenumber $(\mathrm{cm}-1)$
Figure 6. IR spectra of rat retina crosssection.

A. Photoreceptor outer segment. B. Outer nuclear layer. 


\section{References}

1. Sommer, A.J.; Katon, J.E. Proceedings of TAPPI Pulping Conference 1993, 2, 543-554.

2. Harthcock, M.A.; Lentz, L.A.; Davis, B.L.; Krishnan, K. Appl. Spectrosc. 1986, 40, 210-214.

3. Lang, P.; Katon, J.E. in Microbeam Analysis (Romig, A.D.; Chambers, W.F. Eds.), 1986, San Francisco Press, San Francisco, CA, 47-49.

4. Yao, K.H.; Witt, A.F. J. Cryst. Growth 1987, 80, 453-455.

5. Chase, D.B. in The Design, Sample Handling, and Applications of Infrared Microscopes - ASTM STP 949 (Roush, PB, Ed.); ASTM: Philadelphia, 1987, 4-11.

6. Lang, P.L.; Katon, J.E.; O'Keefe, J.F.; Schiering, D.W. Microchem. J. 1986, 34, 319-325.

7. Tungol, M.W.; Bartick, E.G.; Montaser, A. J. Forensic Sci. 1991, 36, 1027-1043.

8. Beauchaine, J.P.; Peterman, J.W.; Rosenthal, R.J. Mikrochim. Acta [Wien] 1988, 1, 133-138.

9. Ryland, S.G. in Practical Guide to Infrared Microspectroscopy (Humecki, HJ, Ed.); Marcel Dekker: New York, 1995, pp 163243.
10. Martoglio, P.A.; Bouffard, S.P.; Sommer, A.J.; Katon, J.E.; Jakes, K.A. Anal. Chem. 1990, 62, 1123a-1128a.

11. Lin, R.; Ritz, G.P. Appl. Spectrosc. 1993, 47, 265-271.

12. Guilhaumou, N.; Dumas, P.; Carr, G.L.; Williams, G.P. Appl. Spectrosc. 1998, 52, 1029-1034.

13. Dong, A., Messerschmidt, R.G.; Reffner, J.A.; Caughy, W.S. Biochem. Biophys. Res. Comm. 1988, 156, 752-756.

14. See various articles contained in Cell. Mol. Biol. 1998, 44.

15. Messerschmidt, R.G. in Microbeam Analysis - 1987 (Geiss, R.H. Ed.); San Francisco Press: San Francisco, CA, 1987, pp 169-172.

16. Messerschmidt, R.G. in Practical Guide to Infrared Microspectroscopy (Humecki, HJ, Ed.); Marcel Dekker: New York, NY, 1995, pp 3-39.

17. Sommer, A.J.; Katon, J.E. Appl. Spectrosc. 1991, 45, 16331640.

18. Messerschmidt, R.G.; Sting, D.W.; Microscope Having Dual Remote Image Masking U.S. Patent 4, 877, 960, 1989.

19. Reffner, J.A.; Vogel, S.H. Microsc. Microanal. (Suppl 2: Proceedings) 1998, 4, 410-411.

20. Reffner, J.A.; Vogel, S.H. Confocal Microspectrometer System, U.S. Patent 5, 864, 139, 1999.

21. Young, P.H. Spectroscopy 1989, 3, 24-30. 